\title{
Neural Activation in Reward Network Associated with Inattentive Symptoms of Attention Deficit Hyperactivity Disorder
}

Max M. Owens ${ }^{\mathrm{a}^{*}}$, James Mackillop ${ }^{\mathrm{a}, \mathrm{b}, \mathrm{c}}$, Shannon McNally ${ }^{\mathrm{a}}$, Iris Balodis ${ }^{\mathrm{b}}$, Lawrence H. Sweet ${ }^{\mathrm{a}, \mathrm{d}}$

${ }^{\text {a }}$ Department of Psychology, University of Georgia, 125 Baldwin Street, Athens, GA 30602

${ }^{b}$ Michael G. DeGroote Centre for Medicinal Cannabis Research and Peter Boris Centre for Addiction Research, St. Joseph's Healthcare Hamilton/McMaster University, 100 West 5th Street, Hamilton, ON L8P 3R2, Canada

${ }^{\mathrm{c}}$ Homewood Research Institute, Riverslea Building, 150 Delhi Street, Guelph, ON N1E 6K9 Canada

${ }^{\mathrm{d}}$ Department of Psychiatry and Human Behavior, Alpert Medical School of Brown University, Box GA1, Providence, Rhode Island 02912

*Corresponding author: Max Owens, email: owensmax03@gmail.com

NOTES: As of 11/16/2018 (date of preprint publication), this draft has not been peer-reviewed and will most likely be changed and revised. Please do not copy or cite without author's permission. The authors welcome any comments or concerns, send correspondence to Max M Owens (owensmax03@gmail.com). 


\begin{abstract}
Attention-deficit/hyperactivity disorder (ADHD) and impulsivity have been linked to the functioning of the brain's reward network. However, many of these studies focus on the anticipatory phase of reward processing and are limited by small sample sizes. In the current study, a community sample of 1081 adults (mean age $=28.8, \mathrm{SD}=3.7)$ completed a computerized functional magnetic resonance imaging task examining reward outcome. Out-ofscanner participants completed self-report measures of ADHD symptoms and impulsivity. A voxelwise t-test of activation during reward outcomes indicated activation in the left and right ventral striatum, the ventromedial prefrontal cortex, and the posterior cingulate, as well as greater activation in sensory and motor areas. In voxelwise regression analyses, neural response to reward in the left striatum, insula, dorsolateral prefrontal cortex, and lateral temporal cortex, as well as bilaterally in the occipital cortex, was inversely associated with inattentive symptoms of ADHD. No associations were found between neural response to reward and hyperactive symptoms of ADHD or impulsivity. Results were generally consistent in follow-up region of interest analyses. These findings suggest activation in reward network regions is linked to inattentive, but not hyperactive symptoms of ADHD, even in those without a diagnosis.
\end{abstract}

Keywords: reward; attention; hyperactive; ADHD; impulsivity; fMRI 


\section{INTRODUCTION}

Attention-deficit/hyperactivity disorder (ADHD) is a psychological disorder characterized by the inability to maintain sustained focused attention, hyperactive behavior, or both (American Psychiatric Association, 2013). The disorder is typically diagnosed in childhood and is often characterized by higher levels of hyperactivity in youth with a shift towards more inattentive symptoms in adulthood (American Psychiatric Association, 2013). One biological factor suspected to underlie ADHD is dysfunction of the dopaminergic reward system, specifically that individuals with ADHD have characteristically less activation in this system (Gallo and Posner, 2016; Plichta and Scheres, 2014; Sagvolden et al., 2005; Sonuga-Barke, 2011; Tripp and Wickens, 2008). This system is made up of a network of brain regions including hubs in the midbrain, striatum, ventromedial prefrontal cortex, and posterior cingulate cortex and is generally understood to be critical to both the anticipation and experience of rewards, though anticipation and experience of reward appear to be differentially related to these hubs, with the striatum being more associated with anticipation of rewards and the cortical regions more associated with experience of reward (Oldham et al., 2018).

Impulsivity is a core component of ADHD (American Psychiatric Association, 2013) that has been independently linked to the dopaminergic reward system (Beck et al., 2009). The term impulsivity is used to refer to a set of dispositions relating to a lack of foresight or planning, which are typically measured through behavioral tasks or self-report (De Wit, 2009; Dick et al., 2010; Whiteside and Lynam, 2001). Thus, the term impulsivity is referencing a large multifactorial construct that has a number of independent neural substrates. According to factor analytic research, these dispositions can broadly be classified into three domains: impulsive choice, impulsive action, and impulsive personality (MacKillop et al., 2016). High levels of these 
dispositions have been shown to be associated with problematic behaviors such as sexual risk taking (Hoyle et al., 2000), credit card debt (Meier and Sprenger, 2010), and addictive disorders (Crews and Boettiger, 2009; MacKillop et al., 2016). While some of these dispositions have been linked previously to reward-related processes in the brain, this is not consistent across all facets of impulsivity and the majority of work conducted in this area has focused on neural activity during reward anticipation rather than reward receipt/outcome (Plichta and Scheres, 2014). For instance, a form of decisional impulsivity delayed reward discounting (DRD), the propensity to devalue future rewards, has been linked to activation in the ventral striatum during anticipation (Hoogman et al., 2013) and receipt (Hariri et al., 2009) of rewards. The Barratt Impulsivity Scale (BIS), a self-report measure of impulsive personality, has also been linked to activation in the ventral striatum during reward receipt (Forbes et al., 2009).

The primary aim of the current study was to clarify the relationship of symptoms of ADHD and impulsivity with an individual's neural response to rewarding outcomes. This was done by measuring the association of fMRI response in regions of the brain known to be activated during rewarding experiences during the receipt of a monetary reward in a card guessing task. This response was then examined for associations with reported symptoms of ADHD and behavioral impulsivity measured by the Continuous Performance Task (CPT) and decisional impulsivity measured by a DRD task. The current study is unique in examining reward outcomes, as the majority of prior research has focused on neural response during anticipation of reward rather than receipt. Additionally, to further parse out ADHD symptoms, the current study separated inattentive and hyperactive symptoms, a distinction that has been made by some, but not all prior studies (see (Plichta and Scheres, 2014). Furthermore, the current study expands upon existing research by testing multiple forms of impulsivity including 
decisional impulsivity in the form of DRD and behavioral inhibition in the form of a continuous performance task (CPT). The data were drawn from the Human Connectome Project, a large publicly available dataset collected and made available by the U.S. National Institutes of Health. The study's hypothesis was that fMRI response in the reward processing network to reward receipt would be negatively associated with ADHD symptoms and positively associated with impulsivity as has been demonstrated with reward anticipation in a previous meta-analysis (Plichta and Scheres, 2014). However, we did not propose specific hypotheses regarding which type of ADHD symptoms or measures of impulsivity would specifically be most associated with reward-induced activity, as prior literature has been inconsistent on these topics. Concurrent with this primary aim, the current study also sought to characterize the response to rewarding outcomes in a large sample of adults whose brain response was collected using state-of-the-art fMRI procedures. While the neural response to reward has been characterized in the past, the current study attempts to replicate and extend findings in a much larger and novel sample that is both well-powered and includes state-of-the-art fMRI methods including multi-slice acquisition allowing for improved temporal and spatial resolution. This is consistent with an increasing desire in the field of neuroimaging to replicate prior fMRI research in larger samples (Poldrack et al., 2017).

\section{METHODS}

\subsection{Participants}

Data were collected from 1206 participants at Washington University in St. Louis over the course of two days as part of the Human Connectome Project between August 2012 and October 2015 and released in full on March 1, 2017. Informed consent was obtained for all participants (consent procedure detailed in (Van Essen et al., 2013a). Participants were 22-35 
years old and had no significant history of psychiatric disorder, substance abuse, neurological disorder or damage, cardiovascular disease, or Mendelian genetic disease (e.g., cystic fibrosis). They also did not have any contraindications for receiving an MRI such as metal devices in the body or claustrophobia. For full details of inclusion and exclusion criteria, see (Van Essen et al., 2013b). 1081 had complete fMRI data for the reward processing task. Demographics of the final sample are reported in Table 1.

30 participants had missing data for one of more covariates and were excluded from analyses testing the association of neural activation with ADHD symptoms and impulsivity. Additionally, 1 participant was excluded for analyses involving the CPT for missing data and 58 participants had been found in a prior study to have invalid DRD data as indicated by having inconsistencies in responding indicating lack of effort or attention to the task (Owens et al., 2017). In order to maximize sample size at all stages, the decision was made to exclude participants from individual analyses pairwise (i.e., only from the analyses requiring the missing data). 
Table 1. Demographic information on final sample.

\begin{tabular}{lc}
\hline & $\begin{array}{c}\text { Final Sample } \\
(\boldsymbol{N}=\mathbf{1 0 8 1})\end{array}$ \\
\hline Sex & \\
Male & $46.0 \%$ \\
Female & $54.0 \%$ \\
Years of Age & $28.8(3.7)$ \\
Race & $75.1 \%$ \\
White or Caucasian & $14.4 \%$ \\
African American & $5.9 \%$ \\
Asian American & $0.2 \%$ \\
Native American & $2.7 \%$ \\
More than one race & $1.7 \%$ \\
Not sure or unknown & \\
Ethnicity & $8.7 \%$ \\
Hispanic or Latino & $90.1 \%$ \\
Not Hispanic or Latino & $1.2 \%$ \\
Not sure or unknown & \\
Income & $7.0 \%$ \\
\$1,000-\$9,999/year & $7.6 \%$ \\
\$10,000-\$19,999/year & $12.7 \%$ \\
\$20,000-\$29,999/year & $11.8 \%$ \\
\$30,000-\$39,999/year & $10.5 \%$ \\
\$40,000-\$49,999/year & $21.2 \%$ \\
\$50,000-\$74,999/year & $13.5 \%$ \\
\$75,000-\$99,999/year & $15.6 \%$ \\
\$100,000-\$149,999/year & $14.9(1.8)$ \\
Years of Education & \\
\hline
\end{tabular}

\subsection{Measures}

Achenbach Self Report Scale

DSM symptoms of ADHD scale of the Achenbach Self Report Scale (ASR; Achenbach, Dumenci, \& Rescorla, 2003) were used as the primary assessment of ADHD symptomology, with one subscale for inattentive symptoms and one for hyperactive symptoms. On this scale, individuals endorse each of 13 symptoms ( 7 for inattentive, 6 for hyperactive) as "Not true" ( 0 ), "Somewhat or sometimes true" (1), or "Very True or Often True" (2). The inattentive symptoms scale had a range from 0 to 14 , mean of $3.2(\mathrm{SD}=2.37), \mathrm{Q} 1$ of 1.00 , median of 3.0, $\mathrm{Q} 3$ of 5, 
maximum of 14, skewness of .89, kurtosis of .91, and Cronbach's $\alpha$ of .71. The hyperactive scale had a range of 0 to 14 , mean of $2.5(\mathrm{SD}=2.0), \mathrm{Q} 1$ of 1 , median of $2, \mathrm{Q} 3$ of 4 , maximum of 12 , skewness of .87, kurtosis of .57, and Cronbach's $\alpha$ of .66.

fMRI Reward Processing Task

The reward processing task was based on a task developed by Delgado et al. (Delgado et al., 2000). In this task participants play a game where they are asked to guess the number on a mystery card to win or lose money. Card numbers range from 1-9 and participants indicate if they think the mystery card number is higher or lower than 5 by pressing one of two buttons on the response box. Feedback is given as the number on the card and either: 1) a green up arrow with " $\$ 1$ " for reward trials, 2) a red down arrow next to $-\$ 0.50$ for loss trials; or 3) gray double headed arrow for neutral trials (i.e., the number "5"). Participants have up to 1.5 seconds to respond, followed by feedback for one second. There is a one second interstimulus interval in which a fixation cross is presented. Thus, one modeled event is 3.5 seconds total (response, feedback, ISI) with the only difference between reward trials and loss trials being the nature of the outcome. A fixed algorithm is used to ensure all participants experience the same number and pattern of wins and losses regardless of their guess (i.e., the task is "fixed"). In each of the two runs, there are two mostly reward (six reward trials pseudo randomly interleaved with either one neutral and one loss trial, two neutral trials, or two loss trials) and two mostly loss (six loss trials interleaved with either one neutral and one reward trial, two neutral trials, or two reward trials) blocks, interleaved with four rest blocks in which participants passively view a fixation cross (15 seconds each). This results in a total of 32 reward events, 32 loss events, and four neutral events. All participants are paid the amount they won in the task (which is the same for all participants). 
This task has been shown to reliably elicit activations in reward related regions (Delgado et al., 2000; Forbes et al., 2009; May et al., 2004; Tricomi et al., 2004).

\section{Functional Magnetic Resonance Imaging Protocol}

fMRI data were collected during the Reward Processing Task using a 32-channel head coil on a 3T Siemens Skyra (Barch et al., 2013). Two 3:12 imaging runs of the task were completed using the following whole-brain echoplanar acquisition parameters: $\mathrm{TR}=720 \mathrm{~ms}$, TE $=33.1 \mathrm{~ms}$, flip angle $=52$ degrees, $\mathrm{FOV}=208 \times 180 \mathrm{~mm}, 722 \mathrm{~mm}$-thick sagittal slices, $2.0 \mathrm{~mm}$ isotropic voxels. A multi-band acceleration factor of 8 was used. Data were preprocessed by HCP scientists using the minimal preprocessing pipeline (Glasser et al., 2013) that includes gradient unwarping, motion correction, field-map based distortion correction, brain boundarybased registration of functional to the structural scan, registration into MNI152 space, and grandmean intensity normalization.

\section{Out-of-Scanner Continuous Performance Task}

Participants were administered the Short Continuous Performance Task (CPT) from the NIH Toolbox (www.nihtoolbox.org) and the University of Pennsylvania Computerized Neuropsychological Testing Battery (Barch et al., 2013). The CPT is a measurement of sustained attention (Gur et al., 2010). Participants were briefly shown a vertical and horizontal red line on a computer screen. In one block, a response is required when the lines form a number and, in another block, they respond when the lines form a letter. Each block consists of 90 stimuli items and lasts 90 seconds. Scores for both total items correct and response rate (milliseconds) were used as measure of vigilance. The task has been validating using functional MRI and factor structure based on neuropsychological and has been shown to be reliable (Moore et al., 2015). The current study used the indices of Sensitivity and Specificity provided in the HCP database. 
CPT Sensitivity is calculated by the number of targets correctly identified (i.e., true positives) divided by the number of targets correctly identified and the number of lures responded to (i.e., false negatives); this index is indicative of the ability to sustain attention. CPT Specificity is calculated by the number of lures correctly not responded to (i.e., true negatives) divided by the number of lures correctly not responded to plus the number of targets missed (false positives); this index represents the ability to inhibit responding. For both indices, higher scores indicate better performance.

\section{Out-of-Scanner Delay Discounting Task}

A DRD task was administered with participants selecting between smaller amounts of money available immediately or larger amounts in the future (e.g., "would you rather have $\$ 40$ today or $\$ 100$ in six months"). This task used an adaptive adjusting-amount approach in which the delay time was held constant and the immediate dollar amount varied on a trial-by-trial basis in accordance with participants' responding (Estle et al., 2006; Green et al., 2007). This task was completed twice, once using a larger delayed amount of \$200 and once using the larger delayed amount of $\$ 40,000$. For each amount, participants' points of indifference were determined for six periods of time: one month, six months, one year, three years, five years, and ten years. These points, defined in units of dollars, represent the immediate amount at which an individual is indifferent between receiving an immediate reward or delay reward (in this case $\$ 200$ or $\$ 40,000)$ for the given period of time and is conceptualized as the subjective value of a given amount of money at a given delay. In turn, a graphical plot of empirical DRD preferences was generated for each participant using their points of indifference. The plotting of these preferences into a curve permits calculation of the area under that curve, which provides a single measure of DRD that is model-free (not reliant on exponential, hyperbolic, or hyperbaloid modelling 
approaches). Greater area represents less steep DRD and less area represents more steep DRD (Myerson et al., 2001). Crohnbach's $\alpha$ was .83 for the 12 indifferences points (6 per delay period) and the area under the curves of $\$ 200$ and $\$ 40,000$ were highly correlated $(r=.66$, $p<.00001)$; as a result, the two measure were averaged into a single measure, heretofore referred to as $D R D$. For this measure, higher scores were indicative of a greater preference for delayed rewards.

\subsection{Data Analysis}

\section{Characterization of Reward Task fMRI Response}

After downloading minimally preprocessed fMRI data from the HCP database, additional fMRI data processing and analysis were then conducted using Analysis of Functional NeuroImages software (AFNI; (Cox, 1996). Data were spatially smoothed using a $6 \mathrm{~mm}$ full width half maximum Gaussian filter. General linear modeling was completed using regressors representing the time course for events in each condition (win trials, loss trials, neutral trials), six nuisance regressors to account for motion, and regressors for linear, quadratic, and cubic trends. As is standard in the literature using the current reward task (Delgado et al., 2000; May et al., 2004; Tricomi et al., 2004), activation during "win" trials (i.e., when $\$ 1.00$ reward was received) was compared to a baseline of activation during "loss" trials (i.e., when participants lost \$.50). Neutral trials, in which participants were told they neither won nor lost, were not used as a baseline as they represented a very small proportion of trials (two neural trials per run, four total). Group-level activation maps aggregating the contrast of win vs. loss trials across all participants were created using one-sample GLMs in 3dttest++. This brainmap was thresholded to $p<1^{-45}$ with a cluster threshold of 20 voxels and empirically defined regions of interest (ROIs) were created from this map. This threshold was substantially more stringent than the 
usually accepted threshold of cluster-corrected $p<.05$ and was used in order to separate core clusters of activation.

Associations of ADHD Symptoms and Impulsivity

Pearson's correlations were conducted to assess the relationship of ADHD symptoms, CPT performance, and DRD.

Voxelwise Analyses of Reward with ADHD Symptoms and Impulsivity

The association of neural response to receipt of reward ADHD inattentive and hyperactive symptoms, CPT Specificity and Sensitivity, and DRD was first tested voxelwise across the brain. To do this, general linear modeling was used to test these associations controlling for all covariate for each voxel. In these analyses covariates used included: age, gender, income, education, race, twin status (MZ or DZ; the HCP cohort is enriched with twins), anxiety symptoms, depression symptoms, and use of tobacco, alcohol, cannabis, or other illicit drugs. In order to reduce the number of comparisons corrected for and reduce the chance of type II error, only voxels that are classified as gray matter in the MNI template were examined. To do this, AFNI's 3dSeg command was used to create a mask of gray matter and all non-gray matter voxels were masked out. To ensure results were not the result of type I error, a cluster-corrected threshold of $p<.01$ was used in voxelwise analyses to account for the five voxelwise tests completed $(.05 / 5=.01)$. To do this, cluster-extent thresholding was completed using the ACF approach in 3dClustSim in AFNI, which indicated a threshold of raw $p<.001$, cluster size $>53$ voxels would achieve cluster-corrected $p<.01$. Of note, the version of $3 \mathrm{dClustSim} / \mathrm{AFNI}$ used includes corrections that have been made to the software in response to concerns of inflated type I error rate in prior versions (Eklund et al., 2016).

Region of Interest Analyses of Reward with ADHD Symptoms and Impulsivity 
To ensure no relevant results were being missed due to overcorrection at the voxelwise level, region of interest (ROI) analyses were also conducted using empirically defined regions of interest selected based on the reward network regions identified in a recent meta-analysis (Oldham et al., 2018) as being consistently activated during reward outcomes: the right and left ventral striatum, ventromedial prefrontal cortex, and posterior cingulate. These ROIs were created empirically from the contrast of reward vs. loss in the current sample, in which clusters were found in these four regions. This was done by creating a mask from the contrast of reward vs. loss, then using AFNI program 3dROIstats to extract the average activation in all voxels within each of the four ROIs. Then using multiple regression in SPSS each of these ROIs was tested for associations with five measures: ADHD inattentive and hyperactive symptoms, CPT Specificity and Sensitivity, and DRD. Separate multiple regressions were utilized for each region and each behavioral measure (for a total of 20 regression models). The same covariates were used in this analysis as were used in voxelwise analyses. To correct for the number of tests completed, a false discovery rate $q<.05$ (Benjamini and Hochberg, 1995) was used correcting for 20 tests.

\section{RESULTS}

\subsection{Characterization of Reward Task fMRI Response}

During win trials relative to loss trials, significant clusters of activation were found in regions expected to be associated with receipt of reward based on prior literature, including in the striatum, ventromedial prefrontal cortex, and posterior cingulate cortex. Additionally, clusters were found in several unexpected regions including the occipital cortex, posterior parietal cortex, parahippocampal gyrus and cerebellum. See Figure 1 and Table 2. 
Table 2. Cluster table of significant activation during reward (relative to baseline of loss).

Thresholded at cluster-corrected $p<.05\left(p<.01^{-45}\right.$ with a minimum cluster threshold of 20 voxels). Size reported in voxels. Coordinates reported in RAI (Talairach) space represents peak activation in the cluster.

\begin{tabular}{lllllll} 
Hemi & Region & Size & X & Y & Z & Peak t-stat \\
\hline Bilateral & Occipital/Posterior Parietal & 7658 & -1 & 81 & 13 & 44.21 \\
left & Striatum & 876 & 15 & -9 & -5 & 31.82 \\
right & Striatum & 665 & -15 & -10 & -6 & 28.83 \\
Bilateral & Posterior Cingulate Cortex & 567 & -1 & 36 & 34 & 18.75 \\
right & Cerebellum & 537 & -28 & 73 & -43 & 18.83 \\
Bilateral & Ventromedial PFC & 270 & 3 & -44 & 7 & 18.51 \\
left & Cerebellum & 139 & 15 & 79 & -42 & 17.63 \\
right & Inferior Occipital & 83 & -31 & 75 & -10 & 20.52 \\
left & Inferior Occipital & 58 & 18 & 42 & -46 & 19.03 \\
left & Parahippocampal Gyrus & 27 & 28 & 50 & 7 & -19.35 \\
right & Cerebellum & 25 & -22 & 39 & -45 & 16.54
\end{tabular}


Figure 1. Group map of activation during reward (reward trials relative to loss trials).

Thresholded at cluster-corrected $p<.05$ (raw $p<1^{-45}$ with a minimum cluster size of 20 voxels). The clusters labelled were used in region of interest analyses. IVS = left ventral striatum; rVS = right ventral striatum; $\mathrm{PCC}=$ posterior cingulate cortex $; \mathrm{vmPFC}=$ ventromedial prefrontal cortex.

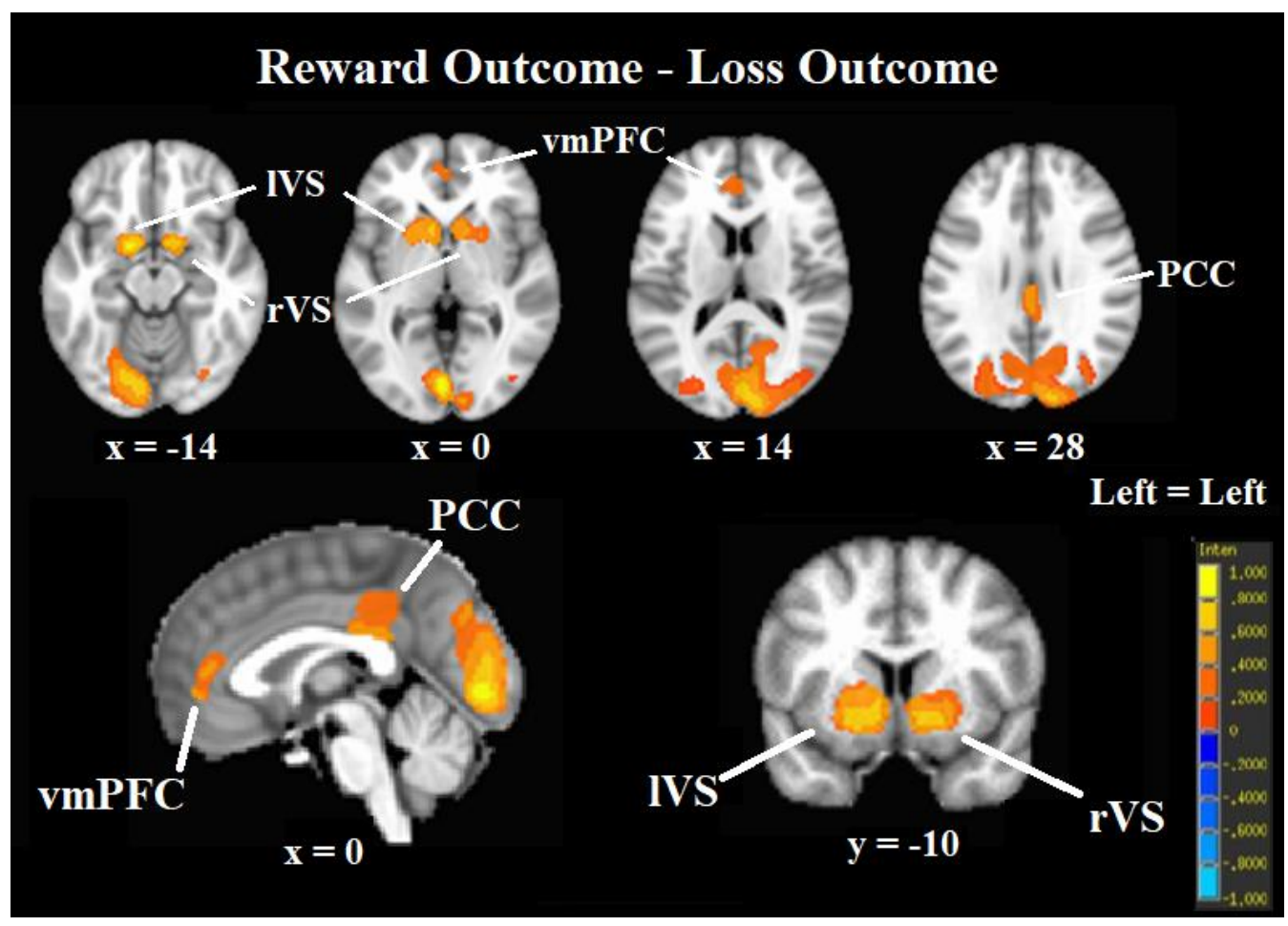

\subsection{Associations of ADHD Symptoms and Impulsivity}

As expected, there was significant, large magnitude correlation between inattentive and hyperactive symptoms (Table 3). CPT Specificity was related to inattentive and hyperactive symptoms. DRD was related to only inattentive symptoms. CPT Sensitivity was not related to inattentive or hyperactive symptoms. 
Table 3. Associations of ADHD symptoms and impulsivity. $* p<.05 ; * * p<.01$.

$\begin{array}{lrrrr} & \text { Inattentive } & \text { Hyperactive } & \text { CPT_SEN } & \text { CPT_SPEC } \\ \text { Hyperactive } & .548^{* *} & & & \\ \text { CPT Sen } & .005 & .012 & & \\ \text { CPT Spec } & -.092^{* *} & -.110^{* *} & .035 & \\ \text { DRD } & .071^{*} & -.002 & .020 & .100^{* *}\end{array}$

\subsection{Voxelwise Analysis}

Significant clusters of association between activation during reward and inattentive symptoms were found in several locations known to be part of the network of regions associated with reward processing (see Table 4; Figure 2). Specifically, clusters were found in the left ventral striatum, left insula, left posterior cingulate, and left middle frontal gyrus. Additionally, clusters were found in several areas associated with visual processing, including the left and right middle occipital cortex and left lingual gyrus. Additionally, several unexpected clusters were found in the lateral temporal cortex, specifically in the superior temporal gyrus, inferior temporal gyrus, and transverse temporal cortex. No clusters were found for the association of reward activation and hyperactive symptoms, CPT sensitivity or specificity, or DRD. 
Table 4. Clusters in which activation during reward (relative to loss) was significantly associated with inattentive symptoms. Coordinates represent location of peak association and are reported in RAI (Talairach) space. Cluster-corrected $p<.05$ (raw $p<.001$, minimum cluster size 53). Size reported in voxels. $\mathrm{Hemi}=$ hemisphere .

\begin{tabular}{llllll} 
Hemi & Region & Size & $\mathbf{X}$ & $\mathbf{Y}$ & $\mathbf{Z}$ \\
\hline left & Insula/Inferior Frontal Gyrus & 506 & 42 & 2 & 14 \\
left & Superior Temporal Gyrus & 317 & 56 & -6 & 0 \\
left & Middle Occipital Gyrus & 314 & 36 & 88 & -2 \\
right & Middle Occipital Gyrus & 215 & -28 & 94 & 2 \\
left & Lingual Gyrus & 168 & 14 & 86 & -18 \\
left & Middle Frontal Gyrus & 163 & 44 & -50 & 0 \\
left & Ventral Striatum & 116 & 24 & -2 & -8 \\
left & Posterior Cingulate Cortex & 113 & 8 & 58 & 4 \\
right & Cuneus & 101 & -10 & 90 & 24 \\
left & Superior Temporal Gyrus & 73 & 48 & 50 & 8 \\
left & Lingual Gyrus & 71 & 4 & 92 & -2
\end{tabular}


Figure 2. Significant associations of activation during reward (relative to loss) with ASR inattentive symptoms. Thresholded at cluster corrected $p<.01($ raw $p<.001$, cluster size $=53)$. IVS = left ventral striatum; PCC = posterior cingulate cortex MFG = middle frontal gyrus; STG $=$ superior temporal gyrus; $\mathrm{mOCC}=$ middle occipital gyrus.

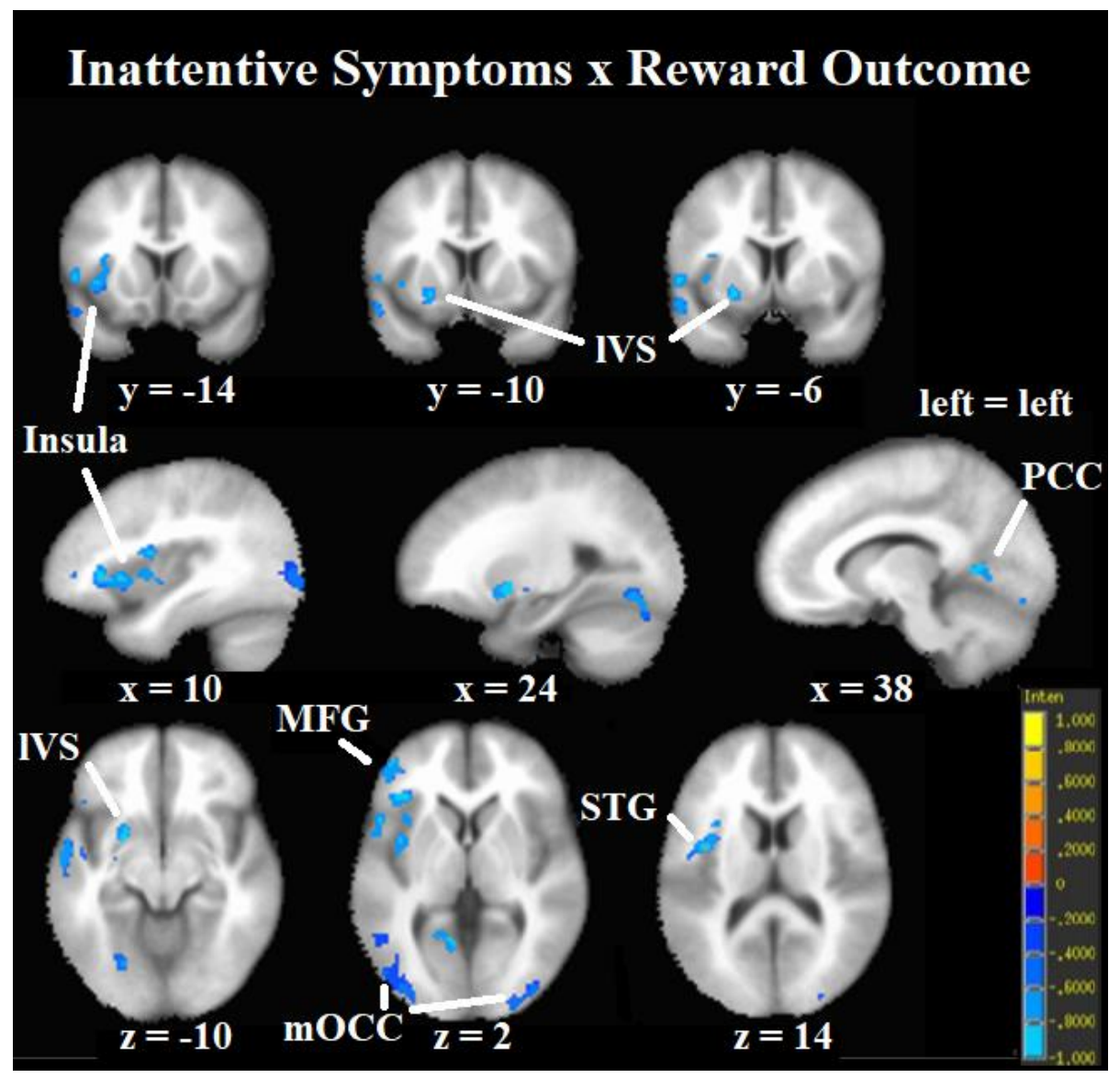

\subsection{Region of Interest Analyses of Reward with ADHD Symptoms and Impulsivity}

Four comparisons were significant beyond false discovery rate correction (Table 5). Inattentive symptoms were associated with reward-related activation in the left striatum and ventromedial prefrontal cortex. Hyperactive symptoms were not associated with reward-related activation in any region of interest. Both $C P T$ sensitivity and $C P T$ specificity were associated with activation in the ventromedial prefrontal cortex. DRD was not associated with rewardrelated activation in any regions. Additionally, activation in the right striatum was nominally 
associated with inattentive symptoms, but did not exceed the False Discovery Rate $q<.05$ threshold set.

Table 5. Results of region of interest analysis. Analyses demonstrate association of activation during reward outcome (relative to during loss outcome) with ADHD symptoms, CPT, and DRD. Results significant beyond FDR $q<.05$ indicated in bold.

\begin{tabular}{clcccc}
\multicolumn{1}{c}{ IV } & Region & $\boldsymbol{\beta}$ & $\boldsymbol{t}$ & $\boldsymbol{p}$ & $\boldsymbol{R}^{2} \boldsymbol{\Delta}$ \\
\hline \multirow{3}{*}{ Inattentive } & Left Ventral Striatum & $\mathbf{- 0 . 1 5}$ & $\mathbf{- 3 . 9 3}$ & $\mathbf{0 . 0 0 0 1}$ & $\mathbf{. 0 1 5}$ \\
Symptoms & Right Ventral Striatum & -0.10 & -2.54 & 0.01 & .006 \\
& Posterior Cingulate Cortex & -0.03 & -0.88 & 0.38 & .001 \\
& Ventromedial Prefrontal Cortex & $\mathbf{- 0 . 1 0}$ & $\mathbf{- 2 . 7 5}$ & $\mathbf{0 . 0 0 6}$ & $\mathbf{. 0 0 7}$ \\
\hline \multirow{3}{*}{ Hyperactive } & Left Ventral Striatum & -0.02 & -0.54 & 0.58 & .000 \\
Symptoms & Right Ventral Striatum & -0.04 & -1.08 & 0.28 & .001 \\
& Posterior Cingulate Cortex & 0.01 & 0.37 & 0.71 & .000 \\
& Ventromedial Prefrontal Cortex & -0.02 & -0.64 & 0.52 & .000 \\
\hline \multirow{5}{*}{ CPT Sensitivity } & Left Ventral Striatum & -0.01 & -0.27 & 0.79 & .000 \\
& Right Ventral Striatum & -0.03 & -1.00 & 0.32 & .001 \\
& Posterior Cingulate Cortex & -0.02 & -0.70 & 0.48 & .000 \\
& Ventromedial Prefrontal Cortex & $\mathbf{- 0 . 0 8}$ & $\mathbf{- 2 . 7 1}$ & $\mathbf{0 . 0 0 7}$ & $\mathbf{. 0 0 7}$ \\
\hline \multirow{5}{*}{ CPT Specificity } & Left Ventral Striatum & 0.05 & 1.50 & 0.13 & .002 \\
& Right Ventral Striatum & 0.06 & 1.79 & 0.07 & .003 \\
& Posterior Cingulate Cortex & 0.06 & 1.78 & 0.08 & .003 \\
& Ventromedial Prefrontal Cortex & $\mathbf{0 . 0 9}$ & $\mathbf{2 . 8 3}$ & $\mathbf{0 . 0 0 5}$ & $\mathbf{. 0 0 8}$ \\
\hline \multirow{5}{*}{ DRD } & Left Ventral Striatum & -0.04 & -1.11 & 0.27 & .001 \\
& Right Ventral Striatum & 0.01 & 0.16 & 0.88 & .000 \\
& Posterior Cingulate Cortex & -0.03 & -0.96 & 0.34 & .001 \\
& Ventromedial Prefrontal Cortex & 0.00 & -0.06 & 0.95 & .000 \\
\hline
\end{tabular}

\section{DISCUSSION}

Results of the current study indicate that inattentive symptoms of ADHD are associated with less neural response to reward outcome in regions of a network associated with of the processing, including the ventral striatum, posterior cingulate, and ventromedial prefrontal cortex. Additionally, inattentive symptoms were associated with less neural response in regions 
associated with attention and self-control, including the middle frontal gyrus and insula (Goulden et al., 2014; Niendam et al., 2012), as well as in regions associated with sensory processing including lateral temporal cortex and occipital cortex (Goodale and Milner, 1992). These findings were particularly concentrated on the left hemisphere, suggesting that the link between neural response to reward and inattentive symptoms may be a primarily left-lateralized effect (among pre-dominantly right-handed individuals). In contrast, hyperactive symptoms of ADHD were not associated with neural response to reward in reward network regions or in any other regions. Additionally, the current results suggest only weak associations between response to reward outcome and impulsivity, with the only significant associations in this domain observed between the ventromedial prefrontal cortex and performance on the CPT. Of note, this association was present for both Sensitivity on the CPT, which is representative of sustained attention, and Specificity on the CPT, which is representative of inhibitory control. However, the direction of these associations were opposite, such that greater neural response to reward outcome was associated with better specificity but poorer sensitivity. Associations of neural response to reward and CPT performance were found only in region of interest analyses and were smaller in effect size than associations of the striatum with inattentive symptoms. DRD was not associated with neural response to reward in any regions.

The finding that subclinical levels of ADHD symptoms are related to response to reward in nodes of the reward network is consistent with prior meta-analytic findings that individuals with diagnosed ADHD demonstrate hypoactivity in this network during anticipation and receipt of reward (Plichta and Scheres, 2014), supporting the extension of pattern to a non-clinical sample. However, in the current study, this effect was specific to inattentive symptoms, not hyperactive symptoms. This is somewhat inconsistent with one study separating inattentive and 
hyperactive symptoms, which found reward anticipation brain activation to be related to hyperactive but not inattentive symptoms (Scheres et al., 2007). One possible reason for this inconsistency is that these study's findings were focused on anticipation of reward rather than receipt of reward. While overlapping, the neural response to reward outcome has been shown to differ from the response to reward anticipation (Oldham et al., 2018) and this may be the reason for the discrepant findings. Therefore, lower levels of activation in the reward network during reward anticipation may be related to hyperactive symptoms and lower activation during reward outcome may be related to inattentive symptoms. Alternately, it is possible that the previous study examined inattentive symptoms and brain response to reward anticipation did not identify an association due lack of power, as it included only 11 individuals with ADHD and 11 controls and the effect sizes reported in the current study were small.

The general lack of a significant relationship between reward-related neural response and impulsivity was somewhat surprising, as prior meta-analytic findings have reported this finding (Plichta and Scheres, 2014). Specifically, DRD was not related to reward outcome activity in any region during voxelwise or region of interest analyses. However, only two prior studies have specifically used DRD as the index of impulsivity, only one of which was on reward outcome (Hariri et al., 2009). Additionally, it was somewhat surprising that the only association of neural response to reward outcome and CPT performance was found in region of interest analyses of the ventromedial prefrontal cortex. While this region is a critical hub related to the experience of reward (particularly receipt of reward), the opposite directionality of associations with Sensitivity and Specificity is difficult to interpret.

In addition to the study's primary results regarding the relationship of ADHD symptoms and impulsivity to neural response to reward, the current study also provides a high-resolution 
mapping of the regions of the brain most associated with reward processing, confirming previous results that the ventral striatum, ventromedial prefrontal cortex, and posterior cingulate cortex represent some of the most critical hubs in the reward processing network of the brain. Results of these analyses also indicate that receipt of reward elicits greater response in sensory and motor areas than other trials which include the same visual and motor stimuli, but do not result in a rewarding outcome. While these findings are consistent with prior research, they do represent one of the largest studies to characterize the neural response to receipt of reward to date, allowing for greater confidence in the accuracy of prior research.

\subsection{Considerations}

One of the most important considerations in the current study is the sample. Unlike the majority of research on the role of reward processing in individuals with ADHD (Plichta and Scheres, 2014), the current study explores symptoms of ADHD in a non-clinical sample. The number of individuals who have been diagnosed with ADHD in the past is not known, as this was neither recorded nor used as an exclusionary criterion. The reason this sample was utilized was its uniquely large size and high-quality imaging methods which allow for the detection of smaller effects than is common in less well-powered studies. If results are generalizable to a clinical sample, it is likely that larger effect sizes would be found.

Beyond the lack of a clinical sample, the current study is also somewhat limited in its assessment of ADHD symptoms. While the ASR is a widely accepted and validated measure that uses items based on the DSM-V criteria of ADHD, it does suffer from the same inherent limitations as all self-report measures of psychopathology, including reporting bias due to possible lack of insight, memory failure, and self-presentations concerns. Future research should 
attempt to confirm these findings using other methods of assessing ADHD symptomology such as clinical interviews, observation, or corroborating reporting of others.

Despite the limitations that must be placed on the interpretation of the results relating to ADHD as a clinical disorder, the current study provides excellent resolution for characterizing the regions of the brain active during reward processing in general. While the regions of the brain that are most implicated in the receipt of reward has been reported previously (Oldham et al., 2018), and given concerns about reproducibility in social science (Ioannidis, 2005) and cognitive neuroscience (Button et al., 2013), this study provides added value by confirming the results of prior research on the neural correlates of reward in one of the largest samples to date. Additionally, the HCP uses novel fMRI techniques that further improve the resolution of this analysis, such as multi-band acceleration which allows for improved temporal and spatial resolution beyond typical fMRI protocols (Boyacioğlu et al., 2015; Preibisch et al., 2015). A further strength of the current study is the two complementary analysis strategies used, with both voxelwise and region of interest approaches being used to limit the risk of type I and II error. These two approaches most clearly converge on the importance of the ventral striatum, which was strongly implicated in both approaches as being the region most clearly associated with inattentive symptoms of ADHD. However, several other regions were detected in only one analysis, suggesting more caution is warranted in the interpretation of the roles of these regions in reward processing in individuals with ADHD symptoms.

\subsection{Conclusions}

The current study provides evidence from a large, non-clinical sample that inattentive symptoms of ADHD are associated with less neural response during receipt of reward in regions thought to be vital to the processing of reward, especially the ventral striatum, as well as regions 
associated with attention and motivational salience (dorsolateral prefrontal cortex and insula). This is consistent with the perspective of ADHD as a disorder of hypoactive reward processing, perhaps provoking a person to seek out greater stimulation. Notably, these findings were in contrast to hyperactive symptoms of ADHD, which were not associated with neural response to receipt to reward. The current study also provides one of the highest resolution characterizations of the neural response to receipt of reward to date, confirming the results of prior research. Future research to explore anticipation and receipt of reward together as it relates to symptoms of ADHD and assess how common treatments for ADHD affect these processes is needed. 


\section{ACKNOWLEDGMENTS}

These data are from the Human Connectome Project, WU-Minn Consortium (Principal Investigators: David Van Essen and Kamil Ugurbil; 1U54MH091657) funded by the 16 NIH Institutes and Centers that support the NIH Blueprint for Neuroscience Research; and by the McDonnell Center for Systems Neuroscience at Washington University in St. Louis. The authors are deeply appreciative to the Human Connectome Project for open access to its data. In addition, the work was supported by the Peter Boris Chair in Addictions Research (JM) and the Gary Sperduto Endowed Professorship in Clinical Psychology (LS). This research did not receive any specific grant from funding agencies in the public, commercial, or not-for-profit sectors. No funding sources were involved in study design or collection, analysis, and interpretation of the data. These findings do not reflect the official position of the National Institutes of Health.

\section{CONFLICTS OF INTEREST}

MMO, SM, and IB have no potential financial conflicts of interest to declare. LHS receives research funding from the National Institutes of Health. JM receives research funding from the National Institutes of Health, the Canadian Institutes of Health Research, and Correctional Service of Canada, and is a principal in BEAM Diagnostics, Inc.

\section{CONTRIBUTIONS}

Study concept was devised by MMO with assistance from JM and LHS. Later consultation was provided by SM and IB on the design, scope, and presentation of the study. Statistical analysis and fMRI data processing was conducted by MMO. Manuscript was primarily written by MMO with feedback provided by SM, IB, JM, and LHS. 


\section{REFERENCES}

American Psychiatric Association, 2013. Diagnostic and Statistical Manual of Mental Disorders, 5th Edition (DSM-5). Diagnostic Stat. Man. Ment. Disord. 4th Ed. TR. 280. https://doi.org/10.1176/appi.books.9780890425596.744053

Barch, D.M., Burgess, G.C., Harms, M.P., Petersen, S.E., Schlaggar, B.L., Corbetta, M., Glasser, M.F., Curtiss, S., Dixit, S., Feldt, C., Nolan, D., Bryant, E., Hartley, T., Footer, O., Bjork, J.M., Poldrack, R., Smith, S., Johansen-Berg, H., Snyder, A.Z., Van Essen, D.C., 2013. Function in the human connectome: Task-fMRI and individual differences in behavior. Neuroimage 80, 169-189. https://doi.org/10.1016/j.neuroimage.2013.05.033

Beck, A., Schlagenhauf, F., Wüstenberg, T., Hein, J., Kienast, T., Kahnt, T., Schmack, K., Hägele, C., Knutson, B., Heinz, A., Wrase, J., 2009. Ventral striatal activation during reward anticipation correlates with impulsivity in alcoholics. Biol. Psychiatry. https://doi.org/10.1016/j.biopsych.2009.04.035

Benjamini, Y., Hochberg, Y., 1995. Controlling the false discovery rate: a practical and powerful approach to multiple testing. J. R. Stat. Soc. 57, 289-300. https://doi.org/10.2307/2346101

Boyacioğlu, R., Schulz, J., Koopmans, P.J., Barth, M., Norris, D.G., 2015. Improved sensitivity and specificity for resting state and task fMRI with multiband multi-echo EPI compared to multi-echo EPI at 7T. Neuroimage. https://doi.org/10.1016/j.neuroimage.2015.06.089

Button, K.S., Ioannidis, J.P.A., Mokrysz, C., Nosek, B.A., Flint, J., Robinson, E.S.J., Munafò, M.R., 2013. Power failure: why small sample size undermines the reliability of neuroscience. Nat. Rev. Neurosci. 14, 365-376. https://doi.org/10.1038/nrn3475

Cox, R.W., 1996. AFNI: software for analysis and visualization of functional magnetic resonance neuroimages. Comput. Biomed. Res. 29, 162-73. https://doi.org/10.1006/cbmr.1996.0014

Crews, F.T., Boettiger, C.A., 2009. Impulsivity, frontal lobes and risk for addiction. Pharmacol. Biochem. Behav. https://doi.org/10.1016/j.pbb.2009.04.018

De Wit, H., 2009. Impulsivity as a determinant and consequence of drug use: A review of underlying processes. Addict. Biol. https://doi.org/10.1111/j.1369-1600.2008.00129.x

Delgado, M.R., Nystrom, L.E., Fissell, C., Noll, D.C., Fiez, J.A., 2000. Tracking the Hemodynamic Responses to Reward and Punishment in the Striatum. J. Neurophysiol. https://doi.org/10.1152/jn.2000.84.6.3072

Dick, D.M., Smith, G., Olausson, P., Mitchell, S.H., Leeman, R.F., O’Malley, S.S., Sher, K., 2010. Understanding the construct of impulsivity and its relationship to alcohol use disorders. Addict. Biol. https://doi.org/10.1111/j.1369-1600.2009.00190.x

Eklund, A., Nichols, T.E., Knutsson, H., 2016. Cluster failure: Why fMRI inferences for spatial extent have inflated false-positive rates. Proc. Natl. Acad. Sci. 113, 7900-7905. https://doi.org/10.1073/pnas.1602413113

Estle, S.J., Green, L., Myerson, J., Holt, D.D., 2006. Differential effects of amount on temporal and probability discounting of gains and losses. Mem. Cognit. 34, 914-928.

https://doi.org/10.3758/BF03193437 
Forbes, E.E., Hariri, A.R., Martin, S.L., Silk, J.S., Moyles, D.L., Fisher, P.M., Brown, S.M., Ryan, N.D., Birmaher, B., Axelson, D.A., Dahl, R.E., 2009. Altered striatal activation predicting real-world positive affect in adolescent major depressive disorder. Am. J. Psychiatry. https://doi.org/10.1176/appi.ajp.2008.07081336

Gallo, E.F., Posner, J., 2016. Moving towards causality in attention-deficit hyperactivity disorder: Overview of neural and genetic mechanisms. The Lancet Psychiatry. https://doi.org/10.1016/S2215-0366(16)00096-1

Glasser, M.F., Sotiropoulos, S.N., Wilson, J.A., Coalson, T.S., Fischl, B., Andersson, J.L., Xu, J., Jbabdi, S., Webster, M., Polimeni, J.R., Van Essen, D.C., Jenkinson, M., 2013. The minimal preprocessing pipelines for the Human Connectome Project. Neuroimage 80, 105124. https://doi.org/10.1016/j.neuroimage.2013.04.127

Goodale, M.A., Milner, A.D., 1992. Separate visual pathways for perception and action. Trends Neurosci. https://doi.org/10.1016/0166-2236(92)90344-8

Goulden, N., Khusnulina, A., Davis, N.J., Bracewell, R.M., Bokde, A.L., McNulty, J.P., Mullins, P.G., 2014. The salience network is responsible for switching between the default mode network and the central executive network: Replication from DCM. Neuroimage 99, 180190. https://doi.org/10.1016/j.neuroimage.2014.05.052

Green, L., Myerson, J., Shah, A.K., Estle, S.J., Holt, D.D., 2007. Do adjusting-amount and adjusting-delay procedures produce equivalent estimates of subjective value in pigeons? J. Exp. Anal. Behav. 87, 337-347. https://doi.org/10.1901/jeab.2007.37-06

Gur, R.C., Richard, J., Hughett, P., Calkins, M.E., Macy, L., Bilker, W.B., Brensinger, C., Gur, R.E., 2010. A cognitive neuroscience-based computerized battery for efficient measurement of individual differences: Standardization and initial construct validation. J. Neurosci. Methods. https://doi.org/10.1016/j.jneumeth.2009.11.017

Hariri, A.R., Gorka, A., Hyde, L.W., Kimak, M., Halder, I., Ducci, F., Ferrell, R.E., Goldman, D., Manuck, S.B., 2009. Divergent Effects of Genetic Variation in Endocannabinoid Signaling on Human Threat- and Reward-Related Brain Function. Biol. Psychiatry. https://doi.org/10.1016/j.biopsych.2008.10.047

Hoogman, M., Onnink, M., Cools, R., Aarts, E., Kan, C., Arias Vasquez, A., Buitelaar, J., Franke, B., 2013. The dopamine transporter haplotype and reward-related striatal responses in adult ADHD. Eur. Neuropsychopharmacol.

https://doi.org/10.1016/j.euroneuro.2012.05.011

Hoyle, R.H., Fejfar, M.C., Miller, J.D., 2000. Personality and sexual risk taking: A quantitative review. J. Pers. https://doi.org/10.1111/1467-6494.00132

MacKillop, J., Weafer, J., C. Gray, J., Oshri, A., Palmer, A., de Wit, H., 2016. The latent structure of impulsivity: impulsive choice, impulsive action, and impulsive personality traits. Psychopharmacology (Berl). https://doi.org/10.1007/s00213-016-4372-0

May, J.C., Delgado, M.R., Dahl, R.E., Stenger, V.A., Ryan, N.D., Fiez, J.A., Carter, C.S., 2004. Event-related functional magnetic resonance imaging of reward-related brain circuitry in children and adolescents. Biol. Psychiatry. https://doi.org/10.1016/j.biopsych.2003.11.008

Meier, S., Sprenger, C., 2010. Present-biased preferences and credit card borrowing. Am. Econ. 
J. Appl. Econ. 2, 193-210. https://doi.org/10.1257/app.2.1.193

Moore, T.M., Reise, S.P., Gur, R.E., Hakonarson, H., Gur, R.C., 2015. Psychometric properties of the penn computerized neurocognitive battery. Neuropsychology. https://doi.org/10.1037/neu0000093

Myerson, J., Green, L., Warusawitharana, M., 2001. Area under the curve as a measure of discounting. J. Exp. Anal. Behav. 76, 235-243. https://doi.org/10.1901/jeab.2001.76-235

Niendam, T.A., Laird, A.R., Ray, K.L., Dean, Y.M., Glahn, D.C., Carter, C.S., 2012. Metaanalytic evidence for a superordinate cognitive control network subserving diverse executive functions. Cogn Affect Behav Neurosci 12, 241-268. https://doi.org/10.3758/s13415-011-0083-5

Oldham, S., Murawski, C., Fornito, A., Youssef, G., Yücel, M., Lorenzetti, V., 2018. The anticipation and outcome phases of reward and loss processing: A neuroimaging metaanalysis of the monetary incentive delay task. Hum. Brain Mapp. 3398-3418. https://doi.org/10.1002/hbm.24184

Owens, M.M., Gray, J.C., Amlung, M.T., Oshri, A., Sweet, L.H., MacKillop, J., 2017. Neuroanatomical foundations of delayed reward discounting decision making. Neuroimage 161. https://doi.org/10.1016/j.neuroimage.2017.08.045

Plichta, M.M., Scheres, A., 2014. Ventral-striatal responsiveness during reward anticipation in $\mathrm{ADHD}$ and its relation to trait impulsivity in the healthy population: A meta-analytic review of the fMRI literature. Neurosci. Biobehav. Rev. https://doi.org/10.1016/j.neubiorev.2013.07.012

Poldrack, R.A., Baker, C.I., Durnez, J., Gorgolewski, K.J., Matthews, P.M., Munafò, M.R., Nichols, T.E., Poline, J.B., Vul, E., Yarkoni, T., 2017. Scanning the horizon: Towards transparent and reproducible neuroimaging research. Nat. Rev. Neurosci. https://doi.org/10.1038/nrn.2016.167

Preibisch, C., Castrillón G., J.G., Bührer, M., Riedl, V., 2015. Evaluation of multiband EPI acquisitions for resting state fMRI. PLoS One. https://doi.org/10.1371/journal.pone.0136961

Sagvolden, T., Johansen, E.B., Aase, H., Russell, V.A., 2005. A dynamic developmental theory of attention-deficit/hyperactivity disorder (ADHD) predominantly hyperactive/impulsive and combined subtypes. Behav. Brain Sci. https://doi.org/10.1017/S0140525X05000075

Scheres, A., Milham, M.P., Knutson, B., Castellanos, F.X., 2007. Ventral Striatal Hyporesponsiveness During Reward Anticipation in Attention-Deficit/Hyperactivity Disorder. Biol. Psychiatry. https://doi.org/10.1016/j.biopsych.2006.04.042

Sonuga-Barke, E.J.S., 2011. ADHD as a reinforcement disorder - Moving from general effects to identifying (six) specific models to test. J. Child Psychol. Psychiatry Allied Discip. https://doi.org/10.1111/j.1469-7610.2011.02444.x

Tricomi, E.M., Delgado, M.R., Fiez, J.A., 2004. Modulation of Caudate Activity by Action Contingency. Neuron. https://doi.org/10.1016/S0896-6273(03)00848-1

Tripp, G., Wickens, J.R., 2008. Research review: Dopamine transfer deficit: A neurobiological theory of altered reinforcement mechanisms in ADHD. J. Child Psychol. Psychiatry Allied 
Discip. https://doi.org/10.1111/j.1469-7610.2007.01851.x

Van Essen, D.C., Smith, S.M., Barch, D.M., Behrens, T.E.J., Yacoub, E., Ugurbil, K., 2013a. The WU-Minn Human Connectome Project: An overview. Neuroimage 80, 62-79. https://doi.org/10.1016/j.neuroimage.2013.05.041

Van Essen, D.C., Ugurbil, K., Auerbach, E., Barch, D., Behrens, T., Bucholz, R., Chang, A., Chen, L., Corbetta, M., Curtiss, S., Della Penna, S., Feinberg, D., Glasser, M., Harel, N., Heath, A., Larson-Prior, L., Marcus, D., Michalareas, G., Moeller, S., Oostenveld, R., Petersen, S., Prior, F., Schlaggar, B., Smith, S., Snyder, A., Xu, J., Yacoub, E., 2013b. The Human Connectome Project: A data acquisition perspective. Neuroimage 62, 2222-2231. https://doi.org/10.1016/j.neuroimage.2012.02.018.The

Whiteside, S.P., Lynam, D.R., 2001. The five factor model and impulsivity: Using a structural model of personality to understand impulsivity. Pers. Individ. Dif. 30, 669-689. https://doi.org/10.1016/S0191-8869(00)00064-7 